\title{
Two cases of catastrophic deterioration and multiple leaflet detachment in Trifecta valves
}

\author{
Nanami Imai ${ }^{1}$, Yuichiro Kaminishi ${ }^{1}$, Toru Tsukada ${ }^{1}$, Motoo Osaka ${ }^{1}$, HIROAKI \\ SAKAMOTO$^{1}$, Bryan Mathis ${ }^{1}$, Yasusuki Suzuki ${ }^{2}$, and Yuji Hiramatsu ${ }^{1}$ \\ ${ }^{1}$ University of Tsukuba \\ ${ }^{2}$ Tsukuba Daigaku Igaku Iryokei
}

June 23, 2021

\begin{abstract}
Reports of early and catastrophic acute structural valve deterioration (SVD) in Trifecta valves (Abbott, St Paul, MN, USA) with multiple leaflet detachment are rare. We encountered two cases of early SVD in Trifecta valves with tears on two leaflets. Both cases presented with acute heart failure because of aortic insufficiency and underwent redo aortic valve replacement; one patient died due to multiple organ failure caused by cardiogenic shock. Durability issues with valves necessitate long-term vigilance in aortic valve replacement patients.
\end{abstract}

Two cases of catastrophic deterioration and multiple leaflet detachment in Trifecta valves

Nanami Imai

Yuichiro Kaminishi, M.D., Ph.D.

Toru Tsukada, M.D.

Motoo Osaka, M.D., Ph.D.

Hiroaki Sakamoto, M.D., Ph.D.

Mathis Bryan James, Ph.D.

Yasuyuki Suzuki*, M.D., Ph.D.

Yuji Hiramatsu, M.D., Ph.D.

Institutions and Affiliations: Department of Cardiovascular Surgery, University of TSUKUBA. 1-1-1 Tennodai, Tsukuba, Ibaraki 305-8575, Japan

Word Count : 1257

*Corresponding Author:

Yasuyuki Suzuki, M.D., Ph.D.

Department of Cardiovascular Surgery

Faculty of Medicine, University of Tsukuba

1-1-1 Tennodai, Tsukuba, Ibaraki 305-8575, Japan, Tel \& Fax (81)29-853-3097,

E-mail:Ysuzuki@md.tsukuba.ac.jp 
Keyword: AVR; Trifecta; SVD; cusp tear; leaflet tear

\section{Introduction:}

Bovine pericardial valves, featuring internally or externally mounted leaflets, are commonly used for aortic valve replacement (AVR) and have a service lifespan of 15 years or more (1)(2). The Trifecta valve (Abbott, St Paul, MN, USA) has externally mounted leaflets that impart excellent hemodynamic performance compared to internal leaflets (3). However, early structural valve deterioration (SVD) has been recently reported in first- and second-generation valves (4)(5)(6). Here, we detail two catastrophic early SVD cases in Trifecta valves with multiple-leaflet tears. Both the patients and his families provided informed consent for the publication of this report.

\section{Patients and Results}

\section{Case 1}

A 79-year-old male with severe aortic stenosis (AS) underwent AVR using a 23-mm, first-generation Trifecta valve in September 2015. Three years later, the patient presented with congestive heart failure, class III NYHA dyspnea, and severe aortic regurgitation (AR). After treatment for heart failure, platelets decreased to $10,000 / \mu \mathrm{l}$, and idiopathic thrombocytopenic purpura was diagnosed, later found to be caused by Helicobacter pylori that was subsequently eradicated using antibiotics plus a proton pump inhibitor. Oral prednisolone and thrombopoietin receptor agonist were given two months before surgery to treat immune thrombocytopenia, with levels rising to $90,000 / \mu \mathrm{l}$ after treatment. An AVR using a 25-mm Inspiris Resilia aortic valve (Edwards Lifesciences LLC, Irvine, CA, USA) and a graft replacement of the ascending aorta with a 30-mm Dacron tube graft was performed. Intrasurgical findings revealed two leaflet tears at the bottom of the left coronary cusp and at the parastent part of the non-coronary cusp (fig.1). Recovery was uneventful and discharge occurred without any complications.

\section{Case 2}

A 63-year-old male with severe aortic stenosis underwent AVR using a $23 \mathrm{~mm}$, second-generation Trifecta valve in March 2018 but, two years later, was urgently admitted with acute heart failure due to severe AR and visible bilateral pulmonary congestion on X-ray (Fig.2). Blood gas analysis revealed acidosis-induced multiple organ failure and extracorporeal membrane oxygenation resuscitation was necessary for cardiogenic shock and loss of consciousness. An urgent AVR (21-mm Inspiris Resilia aortic valve) was performed. Two leaflet detachments were observed at the bottom of the left coronary cusp and parastent of the non-coronary cusp (fig.3). The patient died on the third postoperative day due to multiple organ failure.

\section{Comment}

The Trifecta valve has demonstrated excellent hemodynamic performance, especially for patients with a small aortic annulus, and acceptable durability within the early postoperative period (3). But, according to a recent study, the Trifecta valve had no hemodynamic superiority in maintaining a large valve orifice area over mid- and long-term periods while calcification and leaflet tear parameters were inferior to CEP (4). Some case reports on early leaflet tears in Trifecta valves $(5,6)$ reported that a single leaflet tear may be causative for mild heart failure but two or more tears risks severe and catastrophic heart failure, necessitating an emergency operation (7). Similarly, both of our cases presented with acute dyspnea symptoms linked to rapid ruptures at two leaflets, with the second case especially requiring resuscitation and rapid operation.

Hemodynamics are the crucial aspect of these early leaflet tears. Vriesendorp et al. conducted a 600 million cycle test in vitro with the Trifecta valve as an externally mounted valve and the CEP and Avalus (Medtronic, Minneapolis, MN, USA) valves as internally mounted (8). Of note, the Trifecta valve experienced leaflet tearing at 500 million cycles, corresponding to 10 years in vivo. High-speed camera analysis revealed that leaflets of externally mounted valves wrap around the stent when closing and this motion causes tissue abrasion and eventual tearing whereas leaflets of internally mounted valves are forced against the opposite leaflets, reducing tensile force from the stent. This finding may explain the higher freedom from reintervention 
seen with the CEP internal mount versus the Trifecta and the Mitroflow (LivaNova Group Inc., Vancouver, Canada) external mounts.

We considered transcatheter aortic valve implantation in surgical aortic valve replacement (TAVI in SAVR) for Case 2 due to a high risk of reoperation. However, cardiogenic shock and loss of consciousness forced emergency surgery and precluded TAVI in SAVR due to time constraints. Also, in such cases, the valve area after TAVI in SAVR may be constricted beyond the initial label size and this possibly smaller area could induce high postprocedural gradients or ostial coronary obstruction. Therefore, although Trifecta valves have an excellent size-to-hemodynamic performance ratio, these smaller valves may increase the difficulty of TAVI in SAVR. (9)

Conclusion:

Trifecta valves from any generation may suffer catastrophic SVD with tears on two or more leaflets that cause severe heart failure and require urgent surgery. This durability problem necessitates extreme long-term vigilance after AVR and a re-evaluation of the utility and durability of externally mounted valves.

References:

1. Bourguignon T, Bouquiaux-Stablo AL, Candolfi P, et al. Very long-term outcomes of the CarpentierEdwards Perimount Valve in aortic position. Ann Thorac Surg 2015; 99: 831-7.

2.Minakata K, Tanaka S, Okawa Y, et al. Long-term outcome of the Carpentier-Edwards Pericardial Valve in the aortic position in Japanese Patients. Circ J 2014; 78: 882-889.

3.Phan K, Ha H, Phan S, et al. Early hemodynamic performance of the third generation St Jude Trifecta aortic prosthesis: A systematic review and meta-analysis. J Thorac Cardiovasc Surg. 2015; 149: 15671575.e1-

4.Youngue C, Lopez DC, Soltesz EG, et al. Durability and performance of 2298 Trifecta Aortic Valve Prostheses: A prospensity-matched Analysis. Ann Thorac Surg. 2021; 111; 1198-206.

5.Hamamoto M, Kobayashi T, Ozawa M, Yoshimura K. Pure cusp tear of Trifecta bioprosthesis 2 years after aortic valve replacement. Ann Thorac Cardiovasc Surg. 2017; 23: 157-160.

6.Kaneyuki D, Nakajima H, Asakura T, et al. Early first-generation Trifecta Valve failure: A case series and a review of the literature. Ann Thorac Surg 2020; 109: 86-93.

7.Reyes G, Monguio E, Gomez-Marino MA, et al. Trifecta bioprosthesis sudden-onset three-leaflet detachment. Ann Thorac Surg. 2021; e127-e128.

8.Vriesendorp M, de Lind van Wijngaarden RAF, Rao V, Moront MG, et al. An in vitro comparison of internally versus externally mounted leaflets in surgical aortic bioprosthesis. Interact Cardiovasc Thorac Surg. 2020; 30: 417- 423.

9.Dvir D, Webb JG, Bleiziffer S, et. al. Transcatheter aortic valve implantation in failed bioprosthetic surgical valves. JAMA 2014; 312: 162-170.

Figure Legends

Figure1. Two leaflet tears at the bottom of the left coronary cusp and at the parastent part of the noncoronary cusp. (yellow arrows)

Figure2. Chest X-ray showing bilateral pulmonary congestion.

Figure3. Two leaflet detachments existed at the bottom of the left coronary cusp and parastent of the non-coronary cusp. (yellow arrows)

Abbreviations.

AS; Aortic stenosis 
AR; Aortic regurgitation

LCC; Left coronary cusp

NCC; Non-coronary cusp

SVD; Structural valve deterioration

\section{Hosted file}

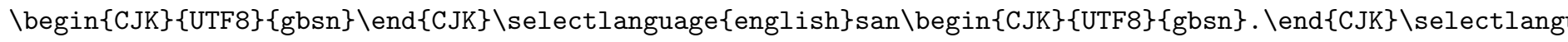
available at https://authorea.com/users/421455/articles/527485-two-cases-of-catastrophicdeterioration-and-multiple-leaflet-detachment-in-trifecta-valves 